\title{
Cardiovascular and Neuroendocrine Responses to Extended Laboratory Challenge
}

Barbara S. McCann, PhD, James Carter, Mary Vaughan, RN, Murray Raskind, MD, Charles W. Wilkinson, PhD, and Richard C. Veith, MD

\begin{abstract}
The purpose of this study was to examine the effects of a 2-hour laboratory challenge on heart rate, blood pressure, catecholamines, and cortisol; and investigate the contribution of the physical act of speaking on both neuroendocrine and cardiovascular measures. Using a within-subjects design, 14 subjects were tested individually during two separate laboratory sessions. During one session, subjects engaged in two cognitively demanding tasks for 2 hours. During the other session, subjects executed the verbal demands of the tasks for 2 hours, but cognitive demands were absent. During both sessions, blood pressure and heart rate were measured and arterialized blood samples were obtained for the measurement of epinephrine, norepinephrine, and cortisol. Subjects demonstrated significantly greater increases in systolic blood pressure, diastolic blood pressure, heart rate, epinephrine, and cortisol during the cognitively challenging session than during the control session. It is concluded that sustained elevations in cardiovascular and neuroendocrine measures can be achieved in the laboratory, and that the effects of such tasks cannot be attributed solely to the physical demands of speaking. Implications for the measurement of circulating catecholamines and cortisol during laboratory studies are discussed.
\end{abstract}

Key words: blood pressure, cortisol, epinephrine, heart rate, laboratory stress, norepinephrine.

\section{INTRODUCTION}

Laboratory studies of cardiovascular and neuroendocrine responses to challenge generally employ tasks of relatively brief duration, typically less than 15 minutes (1). Brief laboratory challenges provoke changes in cardiovascular parameters, such as heart rate and blood pressure, which distinguish people at risk for heart disease from those not at risk (2), differentiate subjects based on individual personality characteristics $(3,4)$, and elucidate relevant task dimensions which impact upon cardiovascular parameters (5).

Despite the heuristic value of brief laboratory challenges in some applications of laboratory methodology, there are several reasons to develop tasks of longer duration (1). This may be particularly true when studying the effects of stress on circulating hormones. Changes in levels of circulating neurohormones often take several minutes to occur, and the administration of brief tasks separated by rest periods of a few minutes may make data interpre-

From the Department of Psychiatry and Behavioral Sciences, University of Washington School of Medicine, and Geriatric Research, Education and Clinical Center, Seattle/American Lake Veterans Administration Medical Centers, Seattle, Washington (M.R., C.W.W., R.C.V).

Address reprint requests to: Barbara S. McCann, PhD, Cardiovascular Behavioral Medicine Program, Psychiatry and Behavioral Sciences, University of Washington School of Medicine, 1100 Olive Way, Suite 1430. Seattle, WA 98101-1827.

Received for publication December 11, 1992; revision received February 25, 1993 tation difficult. For example, it takes approximately 7 minutes from stimulus onset for increases in cortisol to become apparent in the peripheral circulation, and 15 minutes for levels to peak (6).

Extended laboratory tasks may also be needed to more fully explore the cardiovascular effects of stress. It has been shown that the pattern of cardiovascular responses to a laboratory stressor change over the course of 1 hour, presumably reflecting autoregulation. In an extended laboratory session, Miller and Ditto $(7,8)$ showed that during 1 hour of videogame play with shock avoidance, heart rate responses diminished after 15 minutes, although changes in blood pressure and digital blood volume pulse remained fairly constant during the session. However, as these investigators noted, the effect of extended laboratory challenge on neuroendocrine measures has not been studied. Thus, the primary purpose of the present study was to develop a 2hour laboratory protocol and examine its effects on blood pressure, heart rate, catecholamines, and cortisol.

One important consideration in evaluating a longduration laboratory stressor is whether the requirement that subjects speak while engaged in the tasks produces changes in hemodynamic and neuroendocrine measurements because of the physical demands imposed by vocalization, rather than because of the nature of the tasks per se. The physical act of talking has been shown to raise blood pressure (9). However, subsequent research has shown that speaking about a topic of personal relevance results in greater increases in blood pressure and greater decreases in T-wave amplitude and blood volume pulse than does producing neutral speech (10). Sim- 
ilarly, mental arithmetic requiring verbal responses, a cognitively demanding task used frequently in psychophysiological research, has been shown to produce greater increases in heart rate than speaking in a manner which mimics the physical demands of the task without requiring cognitive performance (11). Although cardiovascular measurements (e.g., blood pressure and heart rate) are often accepted as indices of sympathetic nervous system activity, to date no one has examined the contribution of speaking to increases in catecholamines and cortisol. Thus, a second aim of this study was to determine the contribution of the physical act of speaking on both neuroendocrine as well as cardiovascular measures during an extended laboratory session.

A consideration in the execution of the present study was the method of collecting blood samples for catecholamine measurement. Although one typically expects to see increases in circulating levels of epinephrine and norepinephrine in response to stress, several recent investigators have failed to do so (12-14). One possible explanation for the lack of effect on catecholamines in some studies is venous samples have been used, which may reflect local tissue production and removal of norepinephrine and epinephrine, respectively (15). A study comparing arterial and venous sampling at rest and during dental procedures showed that baseline venous levels of norepinephrine are greater than arterial levels; in contrast, baseline venous levels of epinephrine are lower than baseline arterial levels (15). Subsequently, the superiority of arterial measurements over venous ones in examining catecholamine responses to stress has been demonstrated $(16,17)$. Despite the advantages of arterial over venous sampling, the former is considerably more invasive; establishing an intraarterial line is quite painful to the subjects and causes risk of injury. An alternative approach which overcomes many of the limitations of venous sampling is to use arterialized samples, in which a superficial hand vein is used and the limb is heated to increase the rate of arterial-venous exchange. Veith et al. (18) have shown that such "arterialized" sampling is comparable to arterial sampling. Thus, arterialized sampling was used in the present study.

\section{METHODS}

\section{Subjects}

The study protocol was approved by the Human Subjects Review Committee of the University of Washington. Sixteen subjects between the ages of 21 and 65 years were recruited through advertisements seeking healthy men to participate in a study on the effects of stress on heart rate, blood pressure, and stress hormones. Advertisements were posted throughout the Health Sciences Center of the University of Washington. Subjects were paid $\$ 50$ for participating. Data from two subjects were not used because of technical problems with blood collection which arose during their participation. The final sample consisted of 14 nonobese men (aged 21-49 years, mean $=31.8$ ), who were nonsmokers and were not taking medications at the time of study.

\section{Physiological Measures}

Blood pressure and heart rate. Systolic and diastolic blood pressure and heart rate were recorded using an automated oscillometric method (Dinamap, Critikon Co., Tampa, FL).

Catecholamines and cortisol. Blood was collected through a 19. gauge butterfly catheter placed in a dorsal vein of the hand or the cephalic vein of the forearm. The extremity was kept in a warming box thermostatically regulated at $60^{\circ} \mathrm{C}$. This permitted the collection of arterialized venous plasma samples. Each blood sample $(2.5 \mathrm{ml})$ was collected in chilled glass tubes containing EGTA and reduced glutathione and placed on ice. Samples were centrifuged at $4^{\circ} \mathrm{C}$ and plasma was stored at $-70^{\circ} \mathrm{C}$ until assay. Plasma levels of epinephrine and norepinephrine were measured in duplicate by a single isotope enzymatic assay (19). Catechol-omethyltransferase (COMT) enzyme was used for labeling and the catecholamines were separated using thin-layer chromatography (TLC). The intraassay variability is $6 \%$ and interassay variability is $12 \%$ for samples above $100 \mathrm{pg} / \mathrm{ml}$. For samples below $100 \mathrm{pg} /$ $\mathrm{ml}$ the intraassay variability is $10 \%$ and the interassay variability is $15 \%$. All samples from an individual subject were run in the same assay. Cortisol was measured by radioimmunoassay in unextracted plasma samples diluted with phosphate buffer and heated for 20 minutes at $80^{\circ} \mathrm{C}$ to denature binding globulins (20). Cortisol antiserum was obtained from ICM Biomedicals (Costa Mesa, CA). Sensitivity of the assay is $10 \mathrm{pg} / \mathrm{ml}$. Coefficient of variation within assays is $5 \%$ and between assays is less than $10 \%$

\section{Tasks}

Two tasks were used as stressors. The Stroop color-word interference task was presented successively over the course of 1 hour. In this task, subjects were asked to rapidly identify the colors in which words representing colors were printed (e.g., the word "red" printed in the color green, the correct response being "green"). On each successive presentation of this task, subjects were presented with $\mathbf{4 5}$ slides with five words appearing on each slide. Each slide was presented for approximately 5 seconds on a slide projector. Successive administrations of the Paced Auditory Serial Addition Task (PASAT) were presented during the second hour. The PASAT was developed as a measure of the rate of information processing (21). The task consists of four sets of 61 digits each presented on audiotape (numbers 1-9 in random order), in which subjects are asked to add the digits sequentially. The subject is asked to add numbers in pairs aloud, adding each number to the number given before it. For example, given the sequence " $1,9,6$," the correct answers would be " 10 " $(1+9)$ and "15" $(9+6)$. Presentation of the four sets of digits lasts 9 minutes. During the first set, the numbers are presented at a rate of one number every 2.4 seconds. During the subsequent three sets, the 


\section{EXTENDED LABORATORY CHALLENGE}

digits are presented at a rate of every $2.0,1.6$, and 1.2 seconds, respectively.

\section{Procedure}

Subjects were tested individually during two sessions held on separate days. The order of presentation of the Control session and Stress session was counterbalanced. Subjects arrived at the laboratory between 7:00 and 8:00 AM after an overnight fast. After informed consent was obtained, subjects were asked to lay in a hospital bed in the supine position, after voiding. A needle was placed in an appropriate vein for blood collection and was kept patent using a slow infusion of normal saline. Once the needle was in place the hand was positioned in a warming box and the bed was adjusted so that the subject was in a semirecumbent position

After placement of the needle and adjustment of the bed subjects rested quietly for 30 minutes before the start of the tasks. Baseline blood pressure and heart rate measurements, and baseline blood samples were obtained at minutes 25 and 30 .

During the Stress session, the color-word interference task was administered eight times (trials) during the first hour after baseline. Each administration took approximately 5 minutes. A 3 minute intertrial interval occurred between each successive administration of the task. The PASAT was administered five times during the second hour. Each administration of the PASAT took approximately 9 minutes, except for the last administration, which was terminated after 4 minutes. A 5-minute intertrial interval separated each trial. At the end of each intertrial interval during both tasks, the experimenter gave the subject feedback regarding his performance and encouraged him to try to improve his performance on the next trial.

During the Control session, the visual and audiotaped stimuli for the color-word interference task and PASAT were presented to the subject at time intervals identical to presentation during the Stress session. However, the task demands were altered. For the color-word interference task, subjects were instructed to read the words, rather than identify colors. Previous pilot testing in our laboratory had shown that this requires minimal effort on the part of subjects. During the PASAT administration, subjects were instructed to repeat the numbers heard on the audiotape, but no mental arithmetic was required. No performance feedback or encouragement was given to subjects during the intertrial intervals. Instructions during the Control session were to pay close attention in order to read the words and repeat the numbers accurately. These instructions encouraged subjects to attend to the stimuli which were used during the Stress session, and engage in the same amount and rate of vocalization. This insured that the rate of speaking during each session was held constant, because it has been shown previously that faster rates of speaking produce greater increases in blood pressure than slower speaking rates (22).

During the eight administrations of the color-word interference task, over the first hour, blood pressure and heart rate measure ments were obtained at the start of each trial, 2 minutes after the beginning of every other trial, and at the conclusion of every other trial. Blood samples were obtained 2 minutes after the start of four of the eight administrations, corresponding to minutes 10 , 26, 42, and 58 of the first hour. During the five administrations of the PASAT, blood pressure and heart rate measurements were obtained at the beginning of each trial, 6 minutes after the start of each trial, and 2 minutes after the conclusion of each trial Blood samples were obtained 6 minutes after the start of each of the first four trials, corresponding to minutes $6,20,34$, and 48 of the second hour. Subjective ratings of task difficulty, involvement, and arousal were obtained using three 10-point rating scales. The endpoints were labeled "extremely easy-impossible." "completely uninvolved, not trying hard at all-extremely involved, trying as hard as possible," and "completely calm and at ease-extremely excited and aroused." These endpoints were chosen because they have been shown previously to differentiate levels of task difficulty (23). Subjects made ratings of both the color-word interference task and the PASAT during Control and Stress sessions. Ratings were made at the conclusion of each experimental session after the blood pressure cuff and needle were removed.

\section{Data Reduction and Analysis}

Baseline cardiovascular and neuroendocrine measures obtained at minutes 25 and 30 of the initial rest period were averaged to yield a baseline value for each parameter. For each of the three cardiovascular measures, values during each of eight 15-minute periods during each of the two 2-hour sessions were averaged to yield eight period values for each session. Thus, each period reflects an average of start-of-trial, during trial, and endof-trial measurements. For neuroendocrine measures, each of the eight samples obtained during tasks represents a period value. For each of the six dependent measures, the baseline recording for each dependent variable was subtracted from each of the eight period values to yield eight change scores (delta) within respective sessions. The effects of session, period, and order were tested using a 2 (control vs. stress) $\times 8$ (15-minute period) $\times 2$ (order) analysis of variance (ANOVA) on each dependent variable (change from baseline for systolic and diastolic blood pressure, heart rate, epinephrine, norepinephrine, and cortisol). Significance level for the ANOVAs was based on the GreenhouseGeisser correction for degrees of freedom (24).

\section{RESULTS}

\section{Baseline Differences}

Baseline differences between the two sessions were examined using paired $t$ tests for each of the six dependent variables. There were no differences in baseline values between the two sessions for any of the cardiovascular or neuroendocrine parameters $(t<1.75, p>.50)$. Baseline and task values for both sessions are shown in Table 1 .

\section{Task Differences}

Cardiovascular measures. Heart rate. Significant effects of session $(F(1 / 12)=23.42, p<.001)$ and period $(F(7 / 84)=3.94, p<.05)$ were obtained for heart rate. Heart rate was higher during the Stress session than during the Control session. Heart rate diminished over the course of the 2 hours during 
B. S. MCCANN et al.

TABLE 1. Pretask Baseline and Task Means and Standard Deviations of Cardiovascular and Neuroendocrine Measures by Session

\begin{tabular}{|c|c|c|c|c|c|c|}
\hline \multirow{3}{*}{ Session } & \multicolumn{6}{|c|}{ Means (SDs in Parentheses) } \\
\hline & \multicolumn{2}{|c|}{ Systolic blood pressure $(\mathrm{mm} \mathrm{Hg})$} & \multicolumn{2}{|c|}{$\begin{array}{l}\text { Diastolic blood pressure (mm } \\
\qquad \mathrm{Hg})\end{array}$} & \multicolumn{2}{|c|}{ Heart rate $(\mathrm{bpm})$} \\
\hline & Baseline & Task & Baseline & Task & Baselıne & Task \\
\hline Control & $122.6(8.5)$ & $123.3(7.4)$ & $70.5(6.5)$ & $72.7(6.0)$ & $61.4(10.2)$ & $60.8(9.2)$ \\
\hline \multirow[t]{3}{*}{ Stress } & $125.7(7.4)$ & $129.1(8.2)^{\star \star}$ & $70.1(7.2)$ & $76.8(6.1)^{\star \star}$ & $61.0(11.7)$ & $67.6(11.1)^{\ldots \ldots}$ \\
\hline & \multicolumn{2}{|c|}{ Epinephrine $(\mathrm{pg} / \mathrm{ml})$} & \multicolumn{2}{|c|}{ Norepinephrıne (pg/ml) } & \multicolumn{2}{|c|}{ Cortisol $(\mu \mathrm{g} / \mathrm{dl})$} \\
\hline & Baseline & Task & Baseline & Task & Baseline & Task \\
\hline Contral & $47.9(10.9)$ & $53.3(161)$ & $167.9(84.7)$ & $198.2(92.1)^{\star \star}$ & $12.25(2.90)$ & $10.21(2.89)^{\star \star}$ \\
\hline Stress & $42.5(16.7)$ & $76.7(31.9)^{\star \star \star}$ & $170.4(91.2)$ & $224.4(100.2)^{\star \star}$ & $11.69(2.31)$ & $11.70(3.73)$ \\
\hline
\end{tabular}

Note: Task vs. baseline, parred $t$ test, ${ }^{\star} p<.05,{ }^{\star \star} p<.01,{ }^{\star \star} p<.001$.

both the Stress and Control sessions (Figure 1). The Session times Period effect was not significant.

Systolic blood pressure. There was a significant effect of session for systolic blood pressure $(F(1 / 12)$ $=20.99, p<.001$ ). Systolic blood pressure was higher during the Stress session than during the Control session. The Session times Period interaction was significant $(F(7,84)=2.51, p<.05)$. Systolic blood pressure increased during the Stress session and decreased during the Control session (Figure 1).

Diastolic blood pressure. As with the other cardiovascular measures, the main effect of session $(F(1)$ $12)=5.78, p<.05$ ) was significant for diastolic blood pressure. In addition, the main effect for Period for diastolic blood pressure was significant $(F(7 / 84)=$ 2.67, $\mathrm{p}<.05$ ). Diastolic blood pressure declined slightly over the course of two hours during both sessions (Figure 1).

Neuroendocrine Measures. Epinephrine. The effect of session was significant for epinephrine $(F(1)$ $12)=23.57, p<.001)$. Epinephrine levels were higher during the Stress session than during the Control session. The period main effect was significant $(F(7,84)=3.02, p<.05)$. Epinephrine concentrations tended to increase over the course of each session, and although this was most marked during the Stress session (Figure 1), the Session times Period interaction was not significant.

Norepinephrine. For norepinephrine, none of the main effects or interactions were significant.

Cortisol. For cortisol, the effect of session was significant $(F(1 / 12)=22.64, p<.001)$, and the effect of period was significant $(F(7 / 84)=4.31, p<.001)$. Plasma cortisol levels were greater during the Stress session than during the Control session, and displayed a tendency to diminish over the course of each session (Figure 1). There was a significant Ses- sion $\times$ Order interaction for cortisol $(F(1,12)=5.76$, $p<.05)$.

\section{Subjective Ratings}

Subjects' ratings of tasks following the Control session and Stress session are shown in Table 2 . Based on paired $t$ tests, subjects perceived the tasks as more difficult $(t(13)=10.18, p<.001)$, were more involved $(t(13)=11.65, p<.001)$, and were more aroused $(t(13)=11.19, p<.001)$ after the Stress session than following the Control session.

\section{DISCUSSION}

An extended 2-hour laboratory challenge produced greater increases in systolic blood pressure, diastolic blood pressure, heart rate, epinephrine, and cortisol than did a 2-hour control session. Heart rate and diastolic blood pressure decreased over the course of both the 2 hours of laboratory challenge and during the control session. Systolic blood pressure increased during the laboratory challenge, but not during the control session. Plasma epinephrine concentrations increased during both sessions, but was higher during psychological challenge than during the control session. Cortisol diminished over the course of both sessions, but markedly less so during psychological challenge. Although there was a slight diminution in heart rate and diastolic blood pressure during the 2-hour session, both parameters were clearly elevated during psychological challenge relative to the control session. Norepinephrine levels increased significantly above baseline levels during both sessions, but the extent of the increase overall 

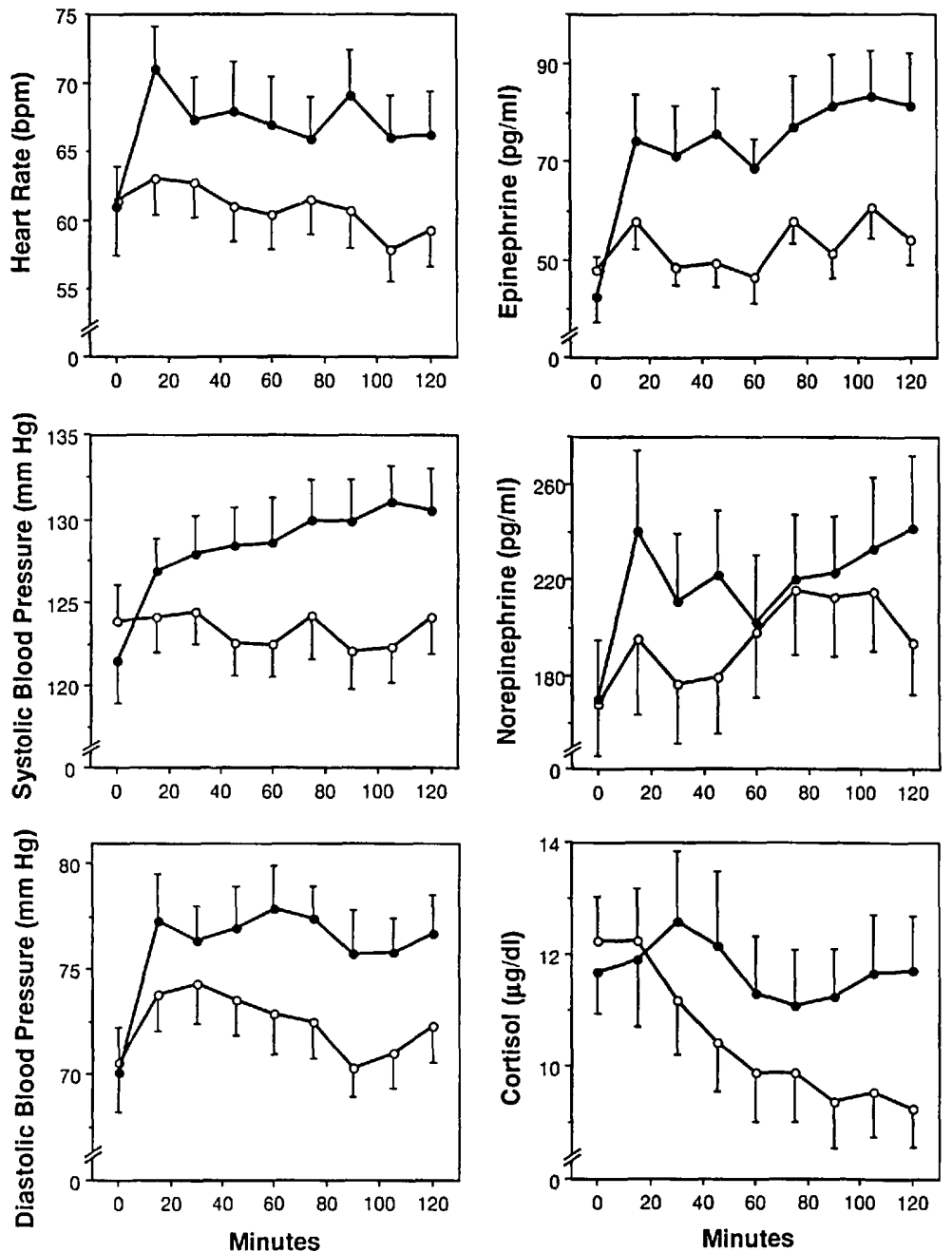

Fig. 1. Mean ( \pm SEM) heart rate, blood pressure, epinephrine, norepinephrine, and cortisol during the Stress session (closed circles) and the Control session (open circles) for each 15-minute period. Time zero represents the baseline value for each variable.

TABLE 2. Average Subject Ratings of Task Dimensions (SD in parentheses)

\begin{tabular}{llc}
\hline \multirow{2}{*}{ Dimensions } & \multicolumn{2}{c}{ Session } \\
\cline { 2 - 3 } & Control & Stress \\
\hline Difficulty & $2.0(0.6)$ & $6.4(1.7)$ \\
Involvement & $3.5(1.4)$ & $8.5(1.0)$ \\
Arousal & $2.8(1.0)$ & $6.7(0.9)$ \\
\hline
\end{tabular}

was not significantly greater during psychological challenge.

An implicit assumption in studies of cardiovascular reactivity in response to brief stressors is that once the stressful task is terminated, blood pressure and heart rate will return to pretask levels following a brief recovery period. In the present study, the small decline in heart rate and diastolic blood pressure which occurred during the administration of 
challenging tasks for 2 hours suggests that it is not merely the absence of stimulation which accounts for "recovery" after participation in a brief task. In the context of extended stress, decrements in heart rate and blood pressure have been attributed to habituation, and are perhaps because of autoregulation $(7,8)$. Interestingly, in our study we observed evidence of habituation in heart rate and diastolic blood pressure although a new stressor was introduced after 1 hour. Thus, it is unlikely that task familiarity accounts for these cardiovascular adjustments, lending additional support to the notion of autoregulation.

Although previous research has shown that the physical act of speaking contributes little to the increases in cardiovascular activity seen during cognitively challenging tasks (11) or affective expression (10), the present study is the first demonstration that speaking in the absence of challenge produces considerably less change in epinephrine and cortisol than active engagement in a difficult task. We found that the physical act of speaking, per se, did not result in a significant increase in epinephrine or cortisol above baseline levels. However, norepinephrine did show an increase above baseline levels during the control session.

The decrease in cortical observed during the control session corresponds to established circadian patterns $(25,26)$. This normal circadian variation in cortisol was offset when subjects were presented with challenging tasks. This pattern is consistent with another recent report that examined the effects of 4 hours of challenging tasks on salivary cortisol (27). These observations highlight the importance of maintaining a consistent starting time for laboratory protocols in which neuroendocrine measures are studied. Additionally, our data attest to the value of taking resting or control measurements during a separate laboratory session conducted at the same time of day as the session in which tasks are administered. Normal circadian variation in cortisol and norepinephrine could override the effects of brief stressors interspersed with rest or recovery periods. Recent failures to see significant increases in these hormones in response to laboratory stress may be due to the timing and frequency of the measurements, reliance on venous samples, or both.

Previous attempts to show an increase in plasma cortisol concentration in response to acute stress have met with mixed results $(28,29)$, despite the assumption that stress activates the pituitary-adrenal system. Levine and Coe (6) have suggested that repeated failures to document increased plasma cortisol levels in the laboratory in response to stressful stimulation may be attributable to inappropriate timing of blood sampling. In particular, they note that obtaining samples at only one or two arbitrary points in time may miss peak changes in cortisol concentration. Our study was able to confirm the effects of acute stress on cortisol levels, probably because of the timing and frequency of measurements and the use of a separate control session on a different day.

The use of arterialized blood samples may account for a clear emergence of epinephrine effects in the present study compared with earlier studies which have relied on venous samples. The determination of plasma catecholamine concentrations based on antecubital venous measurements has many drawbacks $(16,30)$. Forearm tissues extract approximately $50 \%$ of circulating epinephrine, which can result in resting plasma epinephrine concentrations well below the sensitivity of many assay techniques (31). The use of arterialized samples in the present study may have provided the greater sensitivity needed to detect changes in epinephrine concentrations during psychological challenge.

The fact that norepinephrine levels did not show a greater increase during psychological challenge relative to the control session is puzzling, particularly since arterialized samples were used. On the one hand, our findings are consistent with earlier research on catecholamines during stress, in which it was concluded that norepinephrine is not reliably released from sympathetic nerve endings during psychological stress although it increases substantially during physical stress $(32,33)$. On the other hand, subsequent experience from other laboratories in which arterialized samples were used would suggest that our sampling procedure would have produced a difference in norepinephrine levels between the two sessions $(16,17)$. Furthermore, other investigators have recently reported significant increases in norepinephrine during both physical and mental stress using venous sampling (34). However, none of these more recent studies used a separate control session as was used in the present study; thus the extent to which their positive findings could be related to the timing of samples superimposed on diurnal variation is unknown, particularly since plasma norepinephrine tends to increase in the morning (35). In this regard, it is interesting to note that had we restricted the study to examining plasma norepinephrine increases during the tasks in relation to a same-day baseline, we would have reported a significant increase in this hormone (Table 1).

We conclude that an extended protocol is appro- 


\section{EXTENDED LABORATORY CHALLENGE}

priate for studying elevations in cardiovascular and neuroendocrine measures in the laboratory. The use of a separate baseline session may be particularly important. This laboratory paradigm may be useful in studying the time course of stress-induced changes in several plasma constituents of interest to psychosomatic medicine, including various neurohormones and lipids. The extended protocol described herein may be particularly well suited to the study of cortisol responses to stress, because this hormone has a characteristic diurnal variation which may be obscured during laboratory protocols of briefer duration.

This research was supported by National Institutes of Health Grants HL-41657 and AG-08419, and facilitated by the Research Service of the Department of Veteran Affairs. The authors gratefully acknowledge the assistance of Carl Sikkema in analyzing samples for cortisol, Chi Yun for her assistance in the laboratory, and Dr. Patricia Prinz for her comments on an earlier draft of this paper.

\section{REFERENCES}

1. Krantz DS, Manuck SB, Wing RR: Psychological stressors and task variables as elicitors of reactivity. In Matthews KA, Weiss SM, Detre T, Dembroski TM, Falkner B, Manuck SB, Williams RB (eds), Handbook of Stress, Reactivity, and Cardiovascular Disease. New York, John Wiley, 1986, 85-107

2. Stoney CM, Matthews KA: Parental history of hypertension and myocardial infarction predicts cardiovascular responses to behavioral stressors in middle-aged men and women Psychophysiology 25:269-277, 1988

3. Houston BK, Smith MA, Cates DS: Hostility patterns and cardiovascular reactivity to stress. Psychophysiology 26:337342,1989

4. McCann BS, Matthews KA: Influences of potential for hostility. Type A behavior, and parental history of hypertension in adolescents' cardiovascular responses during stress. Psychophysiology 25:503-511, 1988

5. Goldstein IB, Shapiro D: Cardiovascular responses to mental arithmetic and handgrip during different conditions of postural change. Psychophysiology 25:127-136, 1988

6. Levine S, Coe CL: The use and abuse of cortisol as a measure of stress. In Field TM, McCabe PM, Schneiderman N (eds) Stress and Coping. Hillsdale, NJ, Lawrence Erlbaum, 1985, 149-159

7. Miller SB, Ditto B: Cardiovascular responses to an extended aversive video game task. Psychophysiology 25:200-208, 1988

8. Miller SB, Ditto B: Individual differences in heart rate and peripheral vascular responses to an extended aversive task. Psychophysiology 26:506-513, 1989

9. Lynch IJ, Long JM, Thomas SA, et al. The effects of talking on the blood pressure of hypertensive and normotensive individuals. Psychosom Med 43:25-33, 1981

10. Linden W. A microanalysis of autonomic activity during speech. Psychosom Med 49:562-578, 1987
11. Brown TG, Szabo A, Seraganian P: Physical versus psychological determinants of heart rate reactivity to mental arithmetic. Psychophysiology 25:532-537, 1988

12. Davis MC, Matthews KA: Cigarette smoking and oral contraceptive use influence women's lipid, lipoprotein, and cardiovascular responses during stress. Health Psychol 9:717-736, 1990

13. Stoney CM, Owens JF, Matthews KA, et al: Influences of the normal menstrual cycle on physiologic functioning during behavioral stress. Psychophysiology 27:125-135, 1990

14. Sloan RP, Shapiro PA, Gorman JM: Psychophysiological reactivity in cardiac transplant recipients. Psychophysiology $27: 187-194,1990$

15. Halter JB, Pflug AE, Tolas AG: Arterial-venous differences of plasma catecholamines in man. Metabolism 29:9-12, 1980

16. Goldstein DS, Eisenhofer G, Sax FL, et al: Plasma norepinephrine pharmacokinetics during mental challenge. Psychosom Med 49:591-605, 1987

17. Hjemdahl P, Freychuss U, Juhlin-Dannfelt A, Linde B: Differentiated sympathetic activation during mental stress evoked by the Stroop test. Acta Physiol Scand (Suppl) 527:25-29, 1984

18. Veith RC, Best JD, Halter JB: Dose-dependent suppression of norepinephrine appearance rate in plasma by clonidine in man. Clin Endocrinol Metab 59:151-155, 1984

19. Evans MI, Halter JB, Porte D: Comparison of double- and single-isotope derivative methods for measuring catecholamines in human plasma. Clin Chem 24:567-570, 1978

20. Abraham GE, Manlimos FS, Garza R: Radioimmunoassay of steroids. In Abraham GE (ed), Handbook of Radioimmunoassay. New York, Marcel Dekker, 1977, 591-656

21. Gronwall DMA: Paced auditory serial-addition task: A measure of recovery from concussion. Percept Mot Skills 44:367373, 1977

22. Friedmann E, Thomas SA, Kulick-Ciuffo D, et al: The effects of normal and rapid speech on blood pressure. Psychosom Med 44:545-553, 1982

23. Carroll D, Turner JR, Hellawell JC: Heart rate and oxygen consumption during active psychological challenge: The effects of level of difficulty. Psychophysiology 23:174-181, 1986

24. Vasey MW, Thayer JF: The continuing problem of false positives in repeated measures ANOVA in psychophysiology: A multivariate solution. Psychophysiology 24:479-486, 1987

25. De Lacerda L, Kowarski A, Migeon CJ: Integrated concentration and diurnal variation of plasma cortisol. J Clin Endocrinol Metab 36:227-238, 1973

26. Troxler RG, Sprague EA, Albanese RA, et al: The association of elevated plasma cortisol and early atherosclerosis as demonstrated by coronary angiography. Atherosclerosis 26:151162,1977

27. Bohnen N, Nicolson N, Sulon J, Jolles J: Coping style, trait anxiety and cortisol reactivity during mental stress. J Psychosom Res 35:141-147, 1991

28. Goldstein DS, Dionne $R$, Sweet J, et al: Circulatory, plasma catecholamine, cortisol, lipid, and psychological responses to a real-life stress (third molar extractions): Effects of diazepam sedation and of inclusion of epinephrine with the local anesthetic. Psychosom Med 44:259-272, 1982

29. Meyerhoff JL, Oleshansky MA, Mougey EH: Psychologic stress increases plasma levels of prolactin, cortisol, and POMCderived peptides in man. Psychosom Med 50:295-303, 1988

30. Best JD, Halter JB: Release and clearance rates of epinephrine in man: Importance of arterial measurements. J Clin Endocrinol Metab 55:263-268, 1982

31. Veith RC, Murburg MM: Assessment of sympathetic nervous system function in PTSD: A methodologic critique. In MM 


\section{B. S. MCCANN et al.}

Murburg (ed), Catecholamine Function in Post Traumatic Stress Disorder: Emerging Concepts. American Psychiatric Press, Inc., in press

32. Dimsdale JE, Moss J: Plasma catecholamines in stress and exercise. JAMA 243:340-342, 1980

33. Floras J, Vann Jones J. Hassan MO, et al: Failure of plasma norepinephrine to consistently reflect sympathetic activity in humans. Hypertension 8:641-649, 1986
34. Oleshansky MA, Meyerhoff JL: Acute catecholaminergic responses to mental and physical stressors in man. Stress Med 8:175-179, 1992

35. Ziegler MG: Catecholamine measurement in behavioral research. In Schneiderman N, Weiss SM, Kaufmann PG (eds), Handbook of Research Methods in Cardiovascular Behavioral Medicine. New York, Plenum Press, 1989 